\title{
High-Resolution SAR Image Despeckling Based on Nonlocal Means Filter and Modified AA Model
}

\author{
Qiao Ke, ${ }^{1}$ Sun Zeng-guo $\left(\mathbb{D},{ }^{2}\right.$ Yang Liu, ${ }^{3}$ Wei Wei, ${ }^{4}$ Marcin Woźniak (iD, ${ }^{5}$ and Rafał Scherer (iD ${ }^{6}$ \\ ${ }^{1}$ School of Software, Northwestern Polytechnical University, Xi'an 710129, China \\ ${ }^{2}$ School of Computer Science, Shaanxi Normal University, Xi'an 710119, China \\ ${ }^{3}$ College of Computer Science and Technology, Huaqiao University, Xiamen 361021, China \\ ${ }^{4}$ School of Computer Science and Engineering, Xi'an University of Technology, Xi'an 710048, China \\ ${ }^{5}$ Institute of Mathematics, Silesian University of Technology, Kaszubska 23, Gliwice 44-100, Poland \\ ${ }^{6}$ Czestochowa University of Technology, Al. Armii Krajowej 36, Czestochowa 42-200, Poland
}

Correspondence should be addressed to Sun Zeng-guo; sunzg@snnu.edu.cn

Received 7 September 2020; Revised 8 October 2020; Accepted 7 November 2020; Published 29 November 2020

Academic Editor: Manjit Kaur

Copyright (c) 2020 Qiao Ke et al. This is an open access article distributed under the Creative Commons Attribution License, which permits unrestricted use, distribution, and reproduction in any medium, provided the original work is properly cited.

A new speckle suppression algorithm is proposed for high-resolution synthetic aperture radar (SAR) images. It is based on the nonlocal means (NLM) filter and the modified Aubert and Aujol (AA) model. This method takes the nonlocal Dirichlet function as a linear regularization item, which constructs the weight by measuring the similarity of images. Then, a new despeckling model is introduced by combining the regularization item and the data item of the AA model, and an iterative algorithm is proposed to solve the new model. The experiments show that, compared with the AA model, the proposed model has more effective performance in suppressing speckle; namely, ENL and DCV measures are $21.75 \%$ and $4.5 \%$ higher, respectively, than for NLM. Moreover, it also has better performance in keeping the edge information.

\section{Introduction}

Synthetic aperture radar (SAR) is widely used in many aspects, such as ecology, hydrology, ocean monitoring, and topographic mapping for its advantageous of all-day, allweather, multiangle of view, and penetration of ground objects. Because of the coherent imaging system of SAR images, speckle inevitably appears in the imaging process. Especially, imaging of high-resolution SAR images is more complex and demanding. However, the existence of speckle seriously affects the quality of images, makes the interpretation and subsequent processing of images difficult, and cannot correctly reflect the characteristics of the object. Therefore, the speckle suppression in high-resolution SAR images is of great significance [1-3].

There are two main aims of speckle suppression in SAR images. One is to effectively suppress speckle in homogeneous regions, and the other is to preserve edges and fine details as much as possible. SAR image despeckling algorithm has been deeply studied. Traditional filtering algorithms based on local statistics [4] derive local statistics based on homogeneous regions, so the image structure is not preserved enough, the edge is blurred to some extent, and the point target is filtered out. The despeckling method based on wavelet transform [5-7] is the filter based on a fixed window, and image edge information can produce Gibbs phenomenon [8]. In recent years, the method based on partial differential equation (PDE) has become the focus of speckle suppression in SAR images due to its good edge preservation $[9,10]$. Many PDE methods are limited to images with additive noise. Based on this, Aubert and Aujol propose a multiplier speckle suppression model, namely, AA model, in which speckle obeys gamma distribution [11]. The AA model can suppress speckle to a certain extent, but the effect is not very ideal in image edge and texture preservation.

Buades et al. [12] propose a nonlocal means (NLM) filtering algorithm. The algorithm extends the local feature 
statistics of traditional speckle suppression algorithm to nonlocal domain and uses structural similarity to measure the difference between pixels. Compared with traditional filtering algorithm, the NLM filter can preserve image details and texture information well [13-15]. In addition, based on the PDE method, Kindermann et al. [16] propose a general form of energy functional regularization item based on nonlocal means. Some authors use clustering [17] or neural networks [18] for processing satellite images.

Gilboa and Osher [19] construct weights and regularization items by measuring the similarity of images and propose a nonlocal Dirichlet function as a regularization item. In this paper, a new despeckling model of high-resolution SAR images is introduced by combining the regularization item and the data item of AA model, and an iterative algorithm is proposed to solve the new model. Despeckling experiments on different kinds of SAR images demonstrate that, compared with the AA model, the proposed model has more effective performance in suppressing speckle, and it also has better performance in keeping the edge information.

\section{AA Model}

For SAR images, let $u$ be the restored image, $f$ be the observed image, and $v$ be the speckle. Then,

$$
f=u v \text {. }
$$

Based on the Bayesian framework, assuming that the speckle $v$ obeys Gamma distribution, the probability density function is written as follows:

$$
g_{v}(v)=\frac{L^{L}}{\Gamma(L)} v^{L-1} e^{-L v}
$$

where $L$ denotes the number of looks of images and $\Gamma(\cdot)$ denotes the gamma function. By reasoning, Aubert and Aujol proposed a multiplicative noise denoising model, namely, the AA model, as follows:

$$
\underset{u}{\arg \min }\left(T V(u)+\frac{\lambda}{2} \int_{\Omega} \log u(x)+\frac{f(x)}{u(x)} \mathrm{d} x\right),
$$

where regularization item $T V(u)$ is the total variation of $u$, which guarantees the smoothness of the restored image. Data item $\int_{\Omega} \log u(x)+(f(x) / u(x)) \mathrm{d} x$ is used to ensure that the restored image $u$ retains the main features of the observed image $f$ and $\lambda$ is a scale parameter used to balance the regularization item and the data item.

The AA model is a problem of minimizing the total variation and is solved discretely. The discrete iteration form is obtained as follows:

$$
u_{n+1}=u_{n}+\Delta t \operatorname{div}\left(\frac{\nabla u}{|\nabla u|}\right)-\Delta t \lambda\left(\frac{u_{n}-u_{0}}{u_{n}^{2}}\right),
$$

where $\Delta t$ is the time step and $\operatorname{div}(\cdot)$ is the divergence. Figure 1 shows the despeckling results of the AA model for a real high-resolution SAR image, and the SAR image is acquired by the Sandia National Laboratories in the United States. When the maximum peak signal-to-noise ratio (PSNR) of the speckle suppression image is reached, the iteration is stopped. It can be seen that the AA model suppresses speckle well in homogeneous regions, but the effect is not ideal in image edge and texture preservation, which blurs image edge structure information to a certain extent.

\section{NLM Filter}

Buades et al. proposed a NLM filter [12]. Its basic idea is to open a window centered on each pixel $i$ in the image and use every pixel $j$ in the window to estimate the value of the pixel $i$. The estimation uses two smaller window similarities as evaluation criteria. The centers of the two windows are $i$ and $j$, respectively. And weights are calculated using the Gaussian weighted Euclidean distance between the two windows. When the current pixel is estimated, the weight of the pixel which is similar to the central pixel structure in the local structure is larger, and noise can be effectively removed by the weighted mean. The mathematical expression of the NLM filter is written as follows:

$$
\operatorname{NLM}(u)(i)=\frac{1}{C(i, j)} \sum_{j \in \Omega} w(i, j) u(j)
$$

where $\operatorname{NLM}(u)(i)$ is the weighted filtering result, $w(i, j)=$ $e^{-d(i, j) / h^{2}}$ is a filtering weight, $d(i, j)$ is a similarity distance function of pixels $i$ and $j, h$ is the filtering parameter, $C(i, j)=\sum_{j \in \Omega} w(i, j)$ is a normalized function, and $\Omega$ is a neighborhood size of pixel $i$. Parameters $h$ and $\Omega$ affect the final denoising effect.

The SAR image speckle obeys the multiplicative model, and the NLM filtering algorithm is deduced based on the additive Gaussian noise. Therefore, original SAR images are usually transformed logarithmically before processing them. Figure 2 shows the despeckling results of the NLM filter for a real highresolution SAR image, and SAR images were acquired by the Sandia National Laboratories in the United States. It can be seen that the NLM filter has a weak ability to suppress speckle in homogeneous regions but has stronger ability to preserve edge and point targets than the AA model in texture regions.

\section{Modified Model Based on the NLM Filter and the AA Model}

The above results show that the AA model has more effective performance in suppressing speckle in homogeneous regions, but it has weak performance in keeping the edge information. In contrast, the NLM filter has weak performance in suppressing speckle in homogeneous regions, but it has more effective performance in keeping the edge information. Thus, in this paper, a modified model based on the NLM filter and the AA model is proposed. 


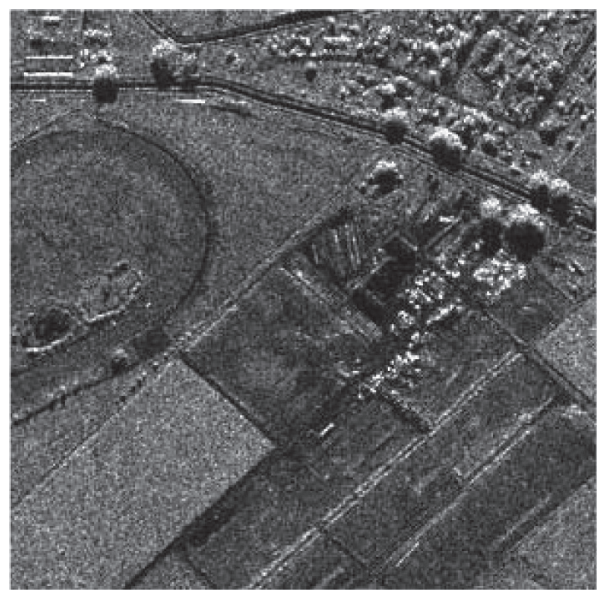

(a)

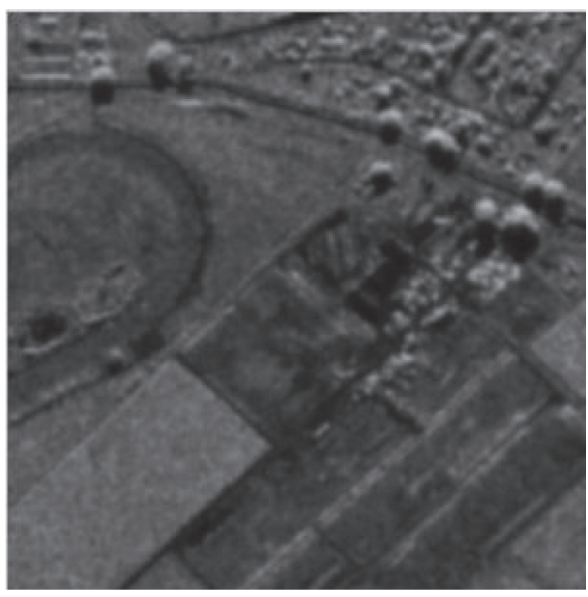

(b)

Figure 1: Despeckling results of SAR image. (a) SAR image; (b) AA model.

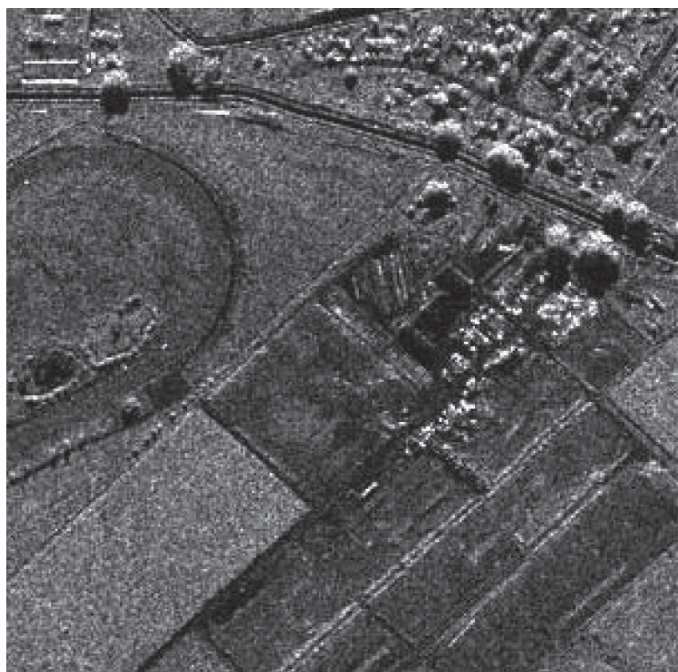

(a)

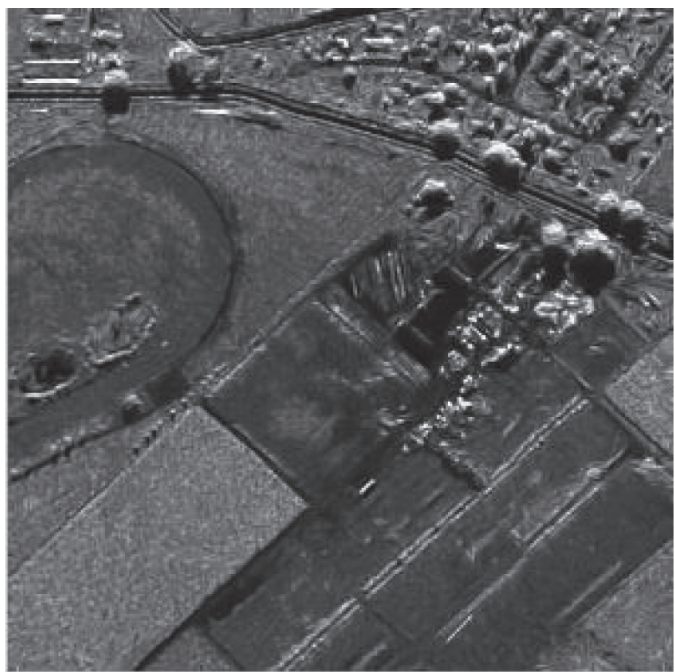

(b)

FiguRE 2: Despeckling results of SAR image. (a) SAR image; (b) NLM filter.

4.1. Establishment of the Model. Gilboa and Osher constructed weights and regularization terms by measuring the similarity of images based on the NLM filter and proposed a nonlocal Dirichlet function as a regularization item:

$$
D(u)=\frac{1}{4} \int_{i \in \Omega}\left|\nabla_{N L} u\right|^{2}(i) \mathrm{d} i=\frac{1}{4} \iint_{\Omega \times \Omega}(u(i)-u(j))^{2} w(i, j) \mathrm{d} i \mathrm{~d} j,
$$

where $w(i, j)=e^{-d(f(i), f(j)) / h^{2}}$ is a weight function, $d(f(i)$, $f(j))$ is a similarity distance function of pixel $i$ and pixel $j$, and $h$ is a filtering parameter. The weight function is used to calculate the similarity between the speckle in the window and the unsuppressed speckle.

In this paper, a modified despeckling model of SAR images is proposed by combining the regularization item and data item of the AA model:

$$
\underset{u}{\arg \min }\left\{\frac{1}{4} \iint_{\Omega \times \Omega}(u(i)-u(j))^{2} w(i, j) \mathrm{d} i \mathrm{~d} j+\frac{\lambda}{2} \int_{\Omega}\left(\log u(i)+\frac{f(i)}{u(i)}\right) \mathrm{d} i\right\} .
$$

4.2. Solution of the Model. Using variational method, the Euler-Lagrange equation corresponding to model (7) is shown as follows:

$$
\int_{\Omega}(u(i)-u(j))(w(i, j)) \mathrm{d} y+\lambda\left(\frac{u(i)-f(j)}{u(i)^{2}}\right)=0 .
$$

Using the steepest descent method, the steepest descent flow is shown as follows: 


$$
u_{t}=\int_{\Omega}(u(i)-u(j)) w(i, j) \mathrm{d} y+\lambda\left(\frac{u(i)-f(j)}{u(i)^{2}}\right)
$$

where scale parameter $\lambda$ can be seen as a Lagrange multiplier and obtained by the following formula:

$$
\lambda=\frac{1}{|\Omega| \sigma^{2}} \int_{\Omega}(u(i)-f(i))\left[\int_{\Omega}(u(j)-u(i)) w(i, j) \mathrm{d} j\right] \mathrm{d} i .
$$

The iteration form of the discretized steepest descending flow (9) is as follows:

$$
u_{i}^{n+1}=u_{i}^{n}+\Delta t \sum_{j} w_{i j}\left(u_{i}^{n}-u_{j}^{n}\right)+\lambda \Delta t \frac{\left(u_{i}^{n}-f_{i}^{0}\right)}{\left(u_{i}^{n}\right)^{2}},
$$

where $u_{i}$ denotes the value of pixel $i, u_{j}$ denotes a pixel value in the neighborhood of pixel $i, w_{i j}$ is the weight function $w(i, j)$, and $\Delta t$ is the time step. In the actual implementation of the algorithm, a small value $\varepsilon$ is added to the denominator to avoid the denominator being zero; then, the discretized iteration form becomes as follows:

$$
u_{i}^{n+1}=u_{i}^{n}+\Delta t \sum_{j} w_{i j}\left(u_{i}^{n}-u_{j}^{n}\right)+\lambda \Delta t \frac{\left(u_{i}^{n}-f_{i}^{0}\right)}{\left(u_{i}^{n}\right)^{2}+\varepsilon} .
$$

The flow chart of the improved filtering algorithm is shown in Figure 3. Firstly, in high-resolution SAR images, pixel $i$ is taken and its neighborhood $\Omega$ and a block to be estimated $N(i)$ are determined. Secondly, in neighborhood $\Omega$ of pixel $i$, the filter weights of $N(i)$ and $N(i)$ are calculated by taking the similar block $N(j)$ of pixel $j$. And then, filter weights of all pixels in the neighborhood and pixel $i$ are calculated by traversing whole neighborhood $\Omega$. Finally, the despeckling result is obtained by putting an iterative formula of the improved model after discretization.

\section{Despeckling Experiments}

Figure 4 shows the despeckling results of a real highresolution SAR image to illustrate the speckle suppression effect of the proposed algorithm. SAR images are acquired by the Sandia National Laboratories in the United States. In order to verify the filtering performance of the proposed algorithm more objectively, some objective evaluation indicators are used to compare the AA model, the NLM filter, and the proposed algorithm. The experiment results are shown in Table 1. An equivalent number of looks (ENL) and difference of the coefficient of variation (DCV) are indexes to evaluate the filtering effect [20-22]. ENL is the most commonly used criterion for SAR image speckle suppression, and its calculation range is homogeneous regions. ENL is defined as the ratio of the square of the mean value of a pixel to the square of the standard deviation in a homogeneous region. The larger the ENL value is, the stronger the speckle suppression ability of the despeckling algorithm is. The calculation range of DCV is edge regions, and it is defined as the difference of the coefficient of variation

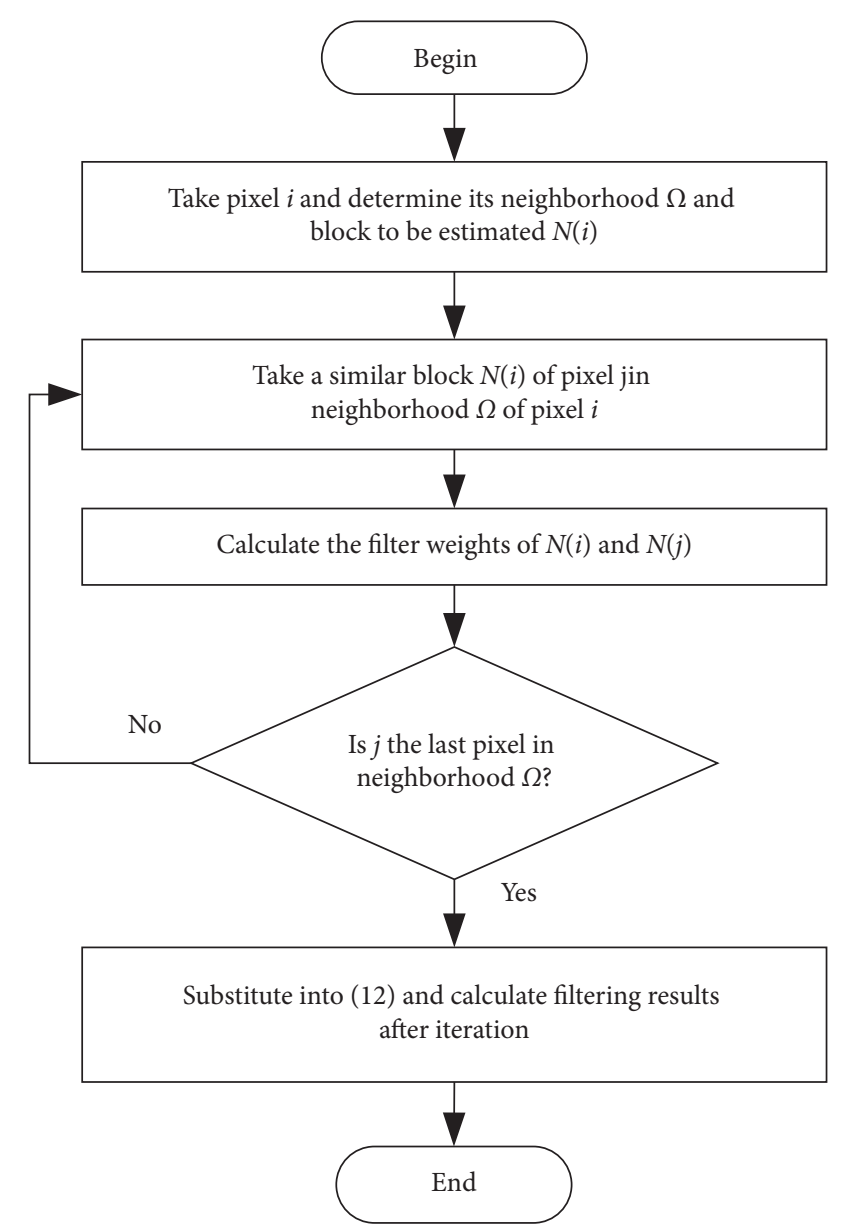

Figure 3: Flow chart of the proposed method.

TABLE 1: Quantitative measures evaluating the performance of various methods in Figure 4.

\begin{tabular}{lcc}
\hline & ENL & DCV \\
\hline AA model & 5.8427 & 0.1935 \\
NLM filter & 3.5489 & 0.0723 \\
Proposed algorithm & 7.8692 & 0.0756 \\
\hline
\end{tabular}

between the real image and speckle suppression image in one edge region. The closer the DCV value is to 0 , the stronger the preservation ability of speckle suppression algorithm for edge information is.

As can be seen from Figure 4, the AA model achieves a better speckle suppression effect in homogeneous regions but largely blurs the edge of the image. In Table 1, the DCV value of the AA model for speckle suppression is the largest, which also confirms that the edge preservation ability of the AA model is the worst. By converting speckle into an additive noise model, the NLM filter is superior to the AA model in preserving edge structure information, and its corresponding DCV value is closer to 0 than that of the AA model. However, the NLM filter has a worse ability to suppress speckle in homogeneous regions than the AA model, and its corresponding ENL value is also smaller than 


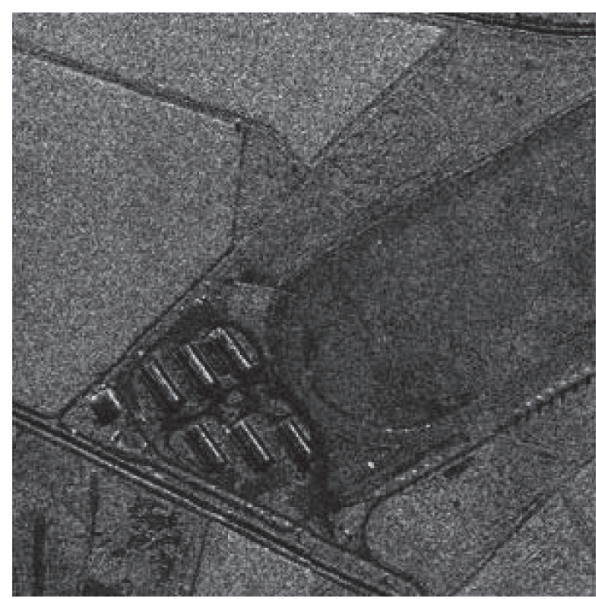

(a)

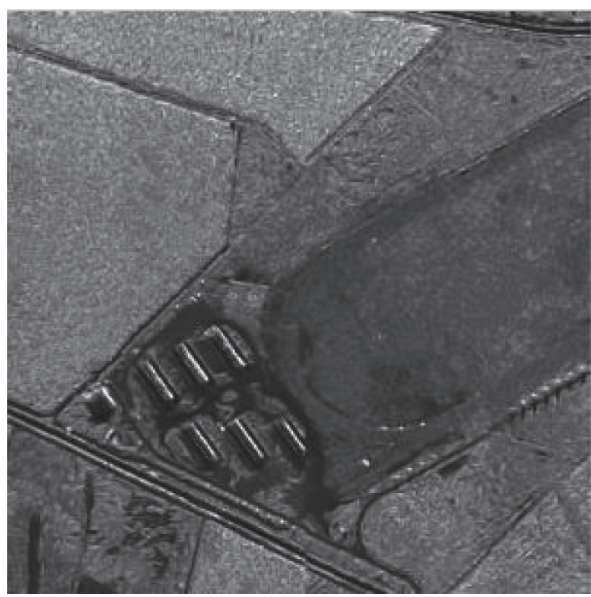

(c)

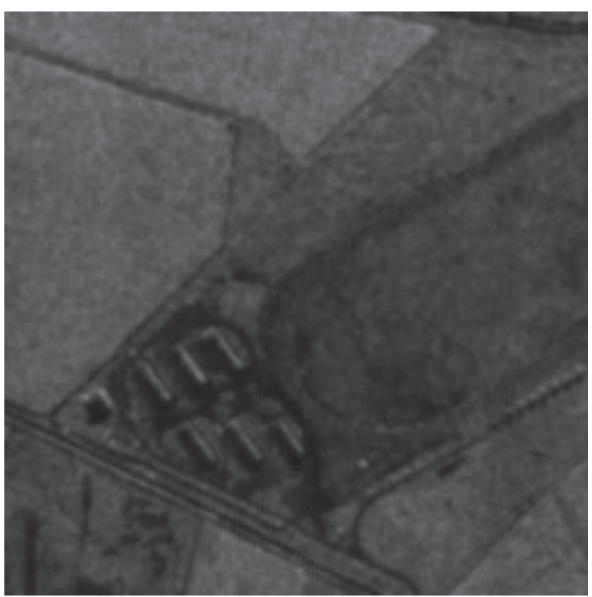

(b)

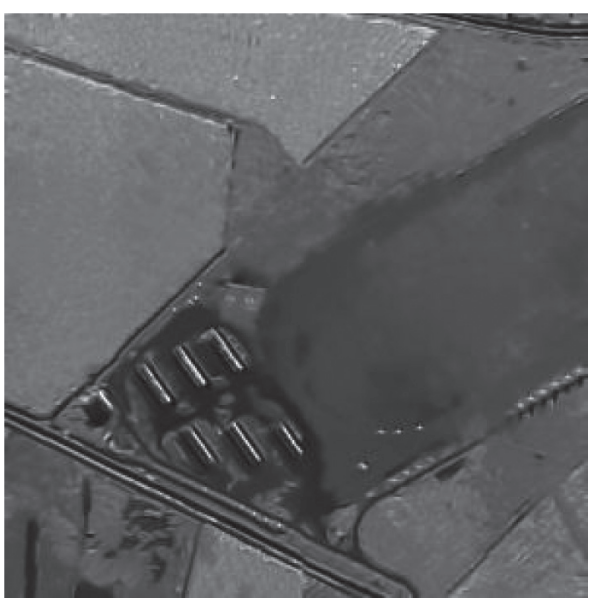

(d)

FIGURE 4: Despeckling results of a SAR image. (a) SAR image; (b) AA model; (c) NLM filter; (d) the proposed method.

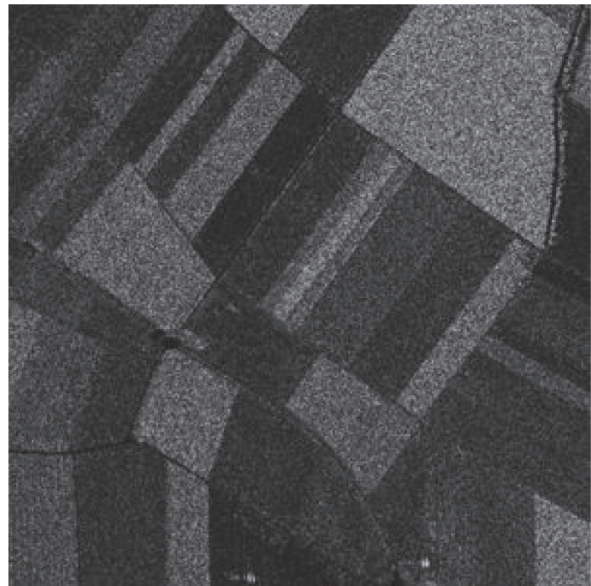

(a)

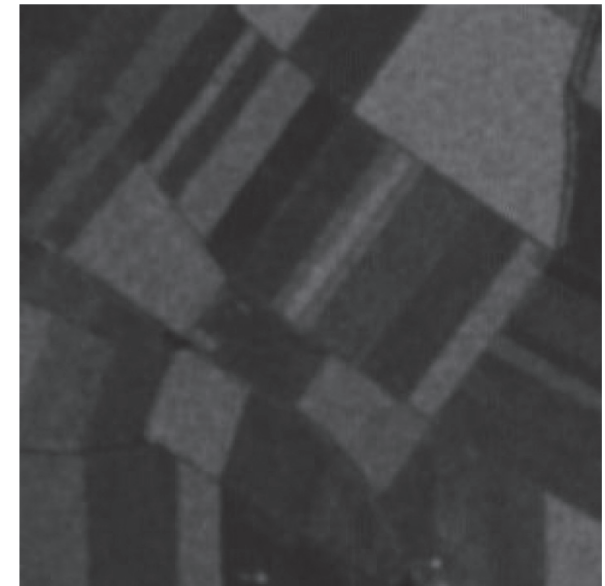

(b)

Figure 5: Continued. 


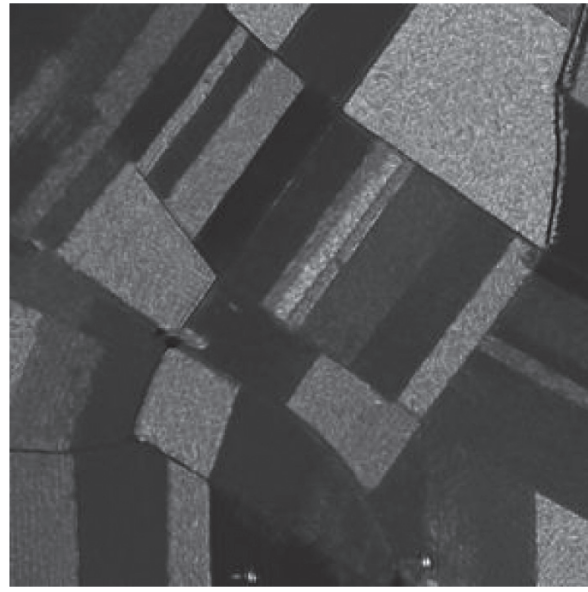

(c)

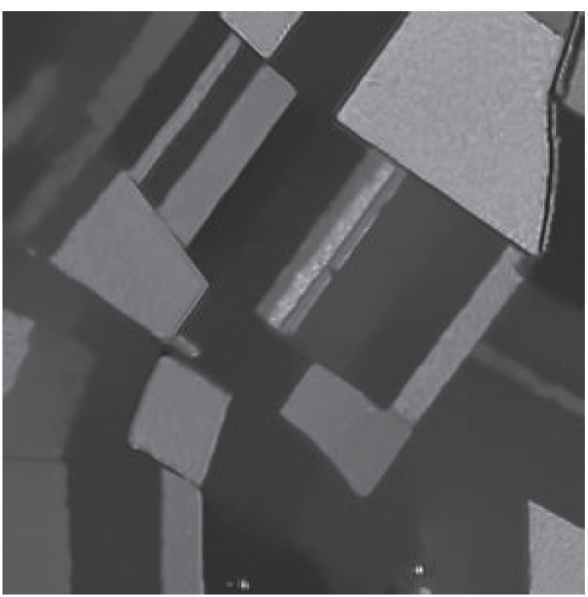

(d)

FIGURE 5: Despeckling results of SAR image. (a) SAR image; (b) AA model; (c) NLM filter; (d) the proposed method.

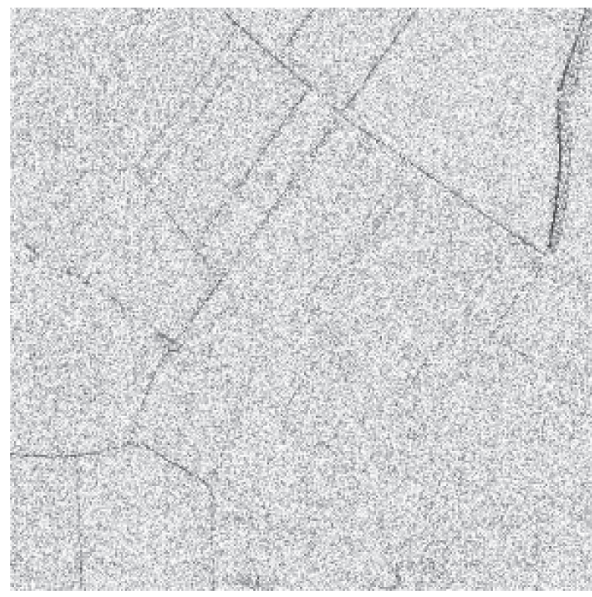

(a)

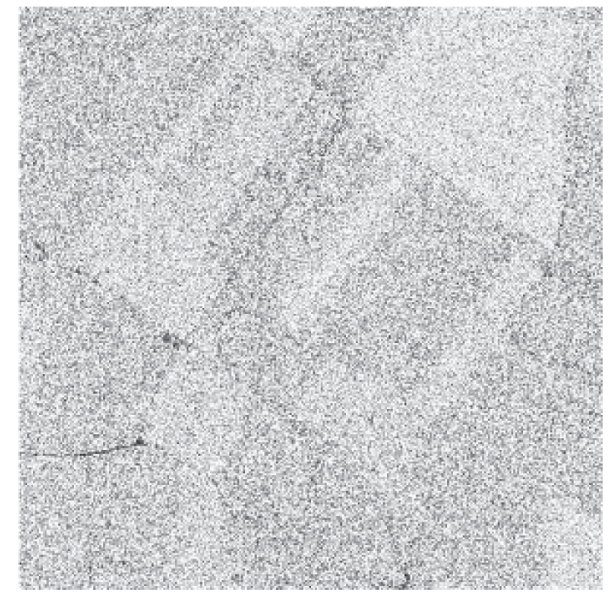

(b)

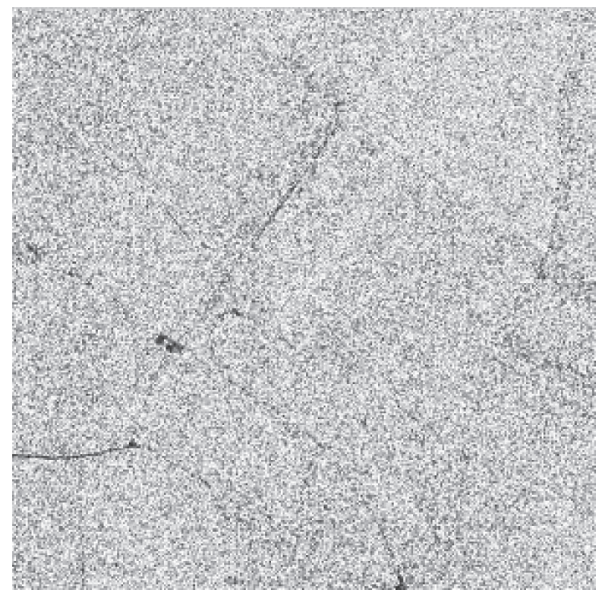

(c)

FIGURE 6: Speckle patterns of various methods in Figure 5. (a) AA model; (b) NLM filter; (c) the proposed method.

that of the AA model. In contrast, the proposed algorithm can suppress speckle in homogeneous regions more thoroughly and preserve edge details better. It can also be seen from Table 1 that the ENL value of the proposed algorithm is the largest, which shows that the proposed algorithm has a more thorough ability of the speckle suppression in 
homogeneous regions than the traditional AA model and the NLM filter. At the same time, the DCV value of the proposed algorithm is closer to 0 than that of the traditional AA model, which shows that the edge preservation ability of the proposed algorithm is stronger.

In order to further illustrate the speckle suppression ability and edge preservation ability of the proposed algorithm for SAR images, the results of the speckle suppression for a SAR image of farmland and the corresponding speckle image are given in Figures 5 and 6, respectively [23-33]. The speckle image is defined by the ratio of the observed image to image after speckle suppression. The amount of edge information on the speckle image reflects the degree of preservation of edge structure information by the speckle suppression method. The less the edge structure information on speckle image, the stronger the edge preservation ability of the corresponding speckle suppression method. The ideal speckle suppression method can completely suppress speckle without losing any edge structure information. In addition, the speckle is not only in homogeneous regions but also in edge regions [33-47].

From the results of the speckle suppression in Figure 5, it can be seen directly that the proposed algorithm is superior to the AA model and the NLM filter in speckle suppression and preserves the edge information of the image. Furthermore, the speckle image in Figure 6 shows that the speckle image corresponding to the proposed algorithm is obviously covered by granular speckle, which shows that the proposed algorithm has better speckle suppression ability than the AA model and the NLM filter. In addition, the AA model has more edge information on the speckle image, while the proposed algorithm has less edge information on the speckle image. This shows that the AA model has a poor edge preservation ability and the proposed algorithm has a strong edge preservation ability.

\section{Conclusions}

In this paper, a new speckle suppression algorithm is proposed for high-resolution SAR images. This method takes the nonlocal Dirichlet function as a linear regularization item, which constructs the weight by measuring the similarity of images. Then, an improved despeckling model is introduced by combining the regularization item and the data item of the AA model, and an iterative algorithm is proposed to solve the new model. Moreover, we compared the proposed method with the AA model and the NLM filter algorithm. The experiments show that the proposed model is more effective in suppressing speckle in homogeneous regions, and it also has a better performance in keeping the edge information. Thus, it turned out to be an effective speckle suppression method for SAR images.

The limitation of our work is a limited number of images. In the future, we plan to perform broader experiments. Besides, we plan to finetune the method parameters.

\section{Data Availability}

No data were used to support this study.

\section{Conflicts of Interest}

The authors declare that they have no conflicts of interest.

\section{Acknowledgments}

The authors would like to thank the US Sandia National Laboratories for providing some SAR images to perform despeckling experiments in this paper. This work was supported by the China Postdoctoral Science Foundation (Nos. 2020TQ0247 and 2020M683567), Development Program of Shaanxi Province (No. 2018ZDXM-GY-036), Shaanxi Key Laboratory of Intelligent Processing for Big Energy Data (No. IPBED7), the Fundamental Research Funds for the Central Universities (No. GK201903085), and the Key Laboratory of Land Satellite Remote Sensing Application Center, Ministry of Natural Resources of the People's Republic of China (No. KLSMNR-202004).

\section{References}

[1] Y.-N. Zhang and L. I. Ying, The Key Technology of SAR Image Processing, Publishing House of Electronics Industry, Beijing, China, 2014.

[2] J. I. A. O. Li-Cheng and B. Hou, Intelligent SAR Image Processing and Interpretation, Science Press, Beijing, China, 2008.

[3] P. I. Yi-Ming, J.-Y. Yang, F. U. Yu-Sheng et al., Synthetic Aperture Radar Imaging Principle, University of Electronic Science and Technology Press, Chengdu, China, 2007.

[4] V. Bhateja, A. Tripathi, A. Gupta et al., An Improved Local Statistics Filter for Denoising of SAR Images, Springer International Publishing, Berlin, Germany, 2014.

[5] R. Tao, H. Wan, and Y. Wang, "Artifact-free despeckling of SAR images using contourlet," IEEE Geoscience and Remote Sensing Letters, vol. 9, no. 5, pp. 980-984, 2012.

[6] J. Ji, X. Li, S.-X. Xu, H. Liu, and J.-J. Huang, "SAR image despeckling by sparse reconstruction based on shearlets," Acta Automatica Sinica, vol. 41, no. 8, pp. 1495-1501, 2015.

[7] S. Lang, X. Liu, B. Zhao, X. Chen, and G. Fang, "Focused synthetic aperture radar processing of ice-sounding data collected over the east antarctic ice sheet via the modified range migration algorithm using curvelets," IEEE Transactions on Geoscience and Remote Sensing, vol. 53, no. 8, pp. 4496-4509, 2015.

[8] J.-J. Xu, SAR Image Despeckling Based on Nonlocal Means Filtering, Xidian University, Xi'an, China, 2010.

[9] Y. Zhao, J. G. Liu, B. Zhang, W. Hong, and Y.-R. Wu, "Adaptive total variation regularization based SAR image despeckling and despeckling evaluation index," IEEE Transactions on Geoscience and Remote Sensing, vol. 53, no. 5, pp. 2765-2774, 2015.

[10] Q. Liu, Z. Yao, and Y. Ke, "Solutions of fourth-order partial differential equations in a noise removal model," Electronic Journal of Differential Equations, vol. 22, no. 3, pp. 249-266, 2007.

[11] G. Aubert and J.-F. Aujol, "A variational approach to removing multiplicative noise," SIAM Journal on Applied Mathematics, vol. 68, no. 4, pp. 925-946, 2008.

[12] A. Buades, B. Coll, and J. M. Morel, "A review of image denoising algorithms, with a new one," Multiscale Modeling \& Simulation, vol. 4, no. 2, pp. 490-530, 2005. 
[13] Y. I. Zi-Lin, D. Yin, and H. U. An-Zhou, "SAR image despeckling based on non-local means filter," Journal of Electronics and Information Technology, vol. 34, no. 4, pp. 950-955, 2012.

[14] Z.-M. Zhao, Y.-J. Zhao, and N. I. U. Chao-Yang, "Improved polarimetric SAR speckle filter based on non-local means," Journal of Image and Graphics, vol. 18, no. 8, pp. 1038-1044, 2013.

[15] Y. Jin, J. Jost, and G. Wang, "A new nonlocal H 1 model for image denoising," Journal of Mathematical Imaging and $\mathrm{Vi}$ sion, vol. 48, no. 1, pp. 93-105, 2014.

[16] S. Kindermann, S. Osher, and P. W. Jones, "Deblurring and denoising of images by nonlocal functionals," Multiscale Modeling \& Simulation, vol. 4, no. 4, pp. 1091-1115, 2005.

[17] G. Capizzi, G. L. Sciuto, M. Woźniak, and R. Damaševičius, "A clustering based system for automated oil spill detection by satellite remote sensing,"Artificial Intelligence and Soft Computing, Berlin, Germany, Springer, pp. 613-623, 2016.

[18] G. Chen, C. Li, W. Wei et al., "Fully convolutional neural network with augmented atrous spatial pyramid pool and fully connected fusion path for high resolution remote sensing image segmentation," Applied Sciences, vol. 9, no. 9, p. 1816, 2019.

[19] G. Gilboa and S. Osher, "Nonlocal linear image regularization and supervised segmentation," Multiscale Modeling \& Simulation, vol. 6, no. 2, pp. 595-630, 2007.

[20] A. Lopes, R. Touzi, and E. Nezry, "Adaptive speckle filters and scene heterogeneity," IEEE Transactions on Geoscience and Remote Sensing, vol. 28, no. 6, pp. 992-1000, 1990.

[21] R. Touzi, "A review of speckle filtering in the context of estimation theory," IEEE Transactions on Geoscience and Remote Sensing, vol. 40, no. 11, pp. 2392-2404, 2002.

[22] A. Lapini, t. Bianchi, F. Argenti, and L. Alparone, "Blind speckle decorrelation for SAR image despeckling," IEEE Transactions on Geoscience and Remote Sensing, vol. 52, no. 2, pp. 1044-1058, 2014.

[23] S. U. N. Zeng-Guo and H. A. N. Chong-Zhao, "Combined despeckling algorithm of synthetic aperture radar images based on region classification, adaptive windowing and structure detection," Acta Physica Sinica, vol. 59, no. 5, pp. 3210-3220, 2010.

[24] P. Zheng, Y. Qi, Y. Zhou, P. Chen, J. Zhan, and M. R.-T. Lyu, "An automatic framework for detecting and characterizing the performance degradation of software systems," IEEE Transactions on Reliability, vol. 63, no. 4, pp. 927-943, 2014.

[25] H. Dou, Y. Qi, W. Wei, and H. Song, "A two-time-scale load balancing framework for minimizing electricity bills of Internet Data Centers," Personal and Ubiquitous Computing, vol. 20, no. 5, pp. 681-693, 2016.

[26] P. Wang, Y. Qi, and X. Liu, "Power-aware optimization for heterogeneous multi-tier clusters," Journal of Parallel and Distributed Computing, vol. 74, no. 1, pp. 2005-2015, 2014.

[27] Y.-n. Qiao, Q. Yong, and H. Di, “Tensor Field Model for higher-order information retrieval," Journal of Systems and Software, vol. 84, no. 12, pp. 2303-2313, 2011.

[28] J. Yan, Y. Qi, and Q. Rao, "Detecting malware with an ensemble method based on deep neural network," Security And Communication Networks, vol. 2018, 16 pages, Article ID7247095, 2018.

[29] X. Wang, Y. Qi, Z. Wang et al., "Design and implementation of SecPod: a framework for virtualization-based security systems," IEEE Transactions on Dependable and Secure Computing, vol. 16, no. 1, pp. 44-57, 2019.

[30] W. Wei and Y. Qi, "Information potential fields navigation in wireless Ad-Hoc sensor networks," Sensors, vol. 11, no. 5, pp. 4794-4807, 2011.
[31] X. Fan, H. Song, X. Fan, and J. Yang, "Imperfect information dynamic stackelberg game based resource allocation using hidden markov for cloud computing," IEEE Transactions on Services Computing, vol. 11, 2018.

[32] H. Song, W. Li, P. Shen, and A. Vasilakos, "Gradient-driven parking navigation using a continuous information potential field based on wireless sensor network," Information Sciences, vol. 408, pp. 100-114, 2017.

[33] Q. Xu, L. Wang, X. H. Hei, P. Shen, W. Shi, and L. Shan, "GI/Geom/1 queue based on communication model for mesh networks," International Journal of Communication Systems, vol. 27, no. 11, pp. 3013-3029, 2013.

[34] Z. Sun, H. Song, H. Wang, and X. Fan, "Energy balance-based steerable arguments coverage method in WSNs," IEEE Access, vol. 99, 2017.

[35] H. Song, H. Wang, and X. Fan, "Research and simulation of queue management algorithms in ad hoc network under DDoS attack," IEEE Access, vol. 5, 2017.

[36] X. Fan, H. Song, and H. Wang, "Video tamper detection based on multi-scale mutual information," Multimedia Tools \& Applications, vol. 78, pp. 1-18, 2019.

[37] X. L. Yang, B. Zhou, J. Feng, and P. Y. Shen, "Combined energy minimization for image reconstruction from few views," Mathematical Problems in Engineering, vol. 2012, Article ID 154630, 15 pages, 2012.

[38] X. L. Yang, P. Y. Shen, and B. Zhou, "Holes detection in anisotropic sensornets: topological methods," International Journal of Distributed Sensor Networks, vol. 8, no. 10, p. 135054, 2012.

[39] W. Wei, Y. Qiang, and J. Zhang, "A bijection between latticevalued filters and lattice-valued congruences in residuated lattices," Mathematical Problems in Engineering, vol. 2013, Article ID 908623, 6 pages, 2013.

[40] H. M. Srivastava, Y. Zhang, L. Wang, P. Shen, and J. Zhang, "A local fractional integral inequality on fractal space analogous to Anderson's inequality," Abstract and Applied Analysis, vol. 2014, Article ID 797561, 14 pages, 2014.

[41] S. Liu, W. Li, and D. Du, "Fractal Intelligent Privacy Protection in Online Social Network Using Attribute-Based Encryption Schemes," IEEE Transactions on Computational Social Systems, vol. 6, no. 3, pp. 736-747, 2019.

[42] X. Fan, M. Woźniak, H. Song, W. Li, Y. Li, and P. Shen, "Ho control of network control system for singular plant," Information Technology And Control, vol. 47, no. 1, pp. 140-150, 2018.

[43] Q. Ke, J. Zhang, H. Song, and Y. Wan, "Big data analytics enabled by feature extraction based on partial independence," Neurocomputing, vol. 288, pp. 3-10, 2018.

[44] J. Zhang, D. P. WeiWei, M. Woźniak, L. Kośmider, and R. Damaševǐcius, "A neuro-heuristic approach for recognition of lung diseases from X-ray images Author links open overlay panel," Expert Systems with Applications, vol. 126, pp. 218-232, 2019.

[45] J. zhang, W. Wei, R. Damasevicius, and M. Wozniak, "Adaptive independent subspace analysis (AISA) of brain magnetic resonance imaging (MRI) data," IEEE Access, vol. 7, no. 1, pp. 12252-12261, 2019.

[46] Q. Ke, J. Zhang, M. Wozniak, and W. Wei, "The phase and shift-invariant feature by adaptive independent subspace analysis for cortical complex cells," Information Technology and Control, vol. 48, 2019.

[47] J. Su, H. Song, H. Wang, and X. Fan, "CDMA-based anticollision algorithm for EPC global C1 Gen2 systems," Telecommunication Systems, vol. 67, no. 3, pp. 1-9, 2018. 